\title{
The Perfect Hyperfluid of Metric-Affine Gravity: The Foundation
}

\author{
Damianos Iosifidis \\ Institute of Theoretical Physics, Department of Physics Aristotle University of Thessaloniki, 54124 Thessaloniki, Greec€
}

(Dated: January 20, 2021)

\begin{abstract}
We set the foundation and formulate the Perfect (Ideal) Hyperfluid. The latter represents the natural generalization of the usual perfect fluid structure where now the microscopic characteristics of matter (spin, shear, dilation) are also taken into account, sourcing a non-Riemannian arena (i.e spacetime torsion and non-metricity) for Metric-Affine Gravity. We derive the energy tensors of this Hyperfluid structure and subsequently present the conservation laws obeyed by them. Finally, we consider a Cosmological application of this Perfect Hyperfluid and classify some possible forms of this fluid structure.
\end{abstract}

\section{Contents}

\section{Introduction}

II. The Setup

III. The sources of Metric-Affine Gravity (MAG)

A. Canonical and Metrical Energy Momentum and Hypermomentum Tensors 3

B. Conservation Laws

IV. Perfect Hyperfluid: Foundation

A. Conservation Laws of the Perfect Hyperfluid

B. Exterior Calculus representation of Perfect Hyperfluid

V. Theories with $\mathcal{L}_{G}=\mathcal{L}_{G}\left(R^{\lambda}{ }_{\alpha \beta \gamma}\right)$

VI. Cosmological Application of the Perfect Hyperfluid

VIII. Acknowledgments

\section{References}

\section{INTRODUCTION}

The Perfect Fluid notion, that we are acquainted with from GR, (or even metric extensions of the latter) has very broad and important applications. However, this collective fluid representation (the Classical Perfect Fluid) is applicable only to matter with no internal structure. At the same time, it is known that in order to probe the nonRiemannian characteristics (torsion and non-metricity) of the underlying spacetime manifold, matter with intrinsic hypermomentum has to be used [1]. It is therefore natural to ask what would be the generalization-extension of the classical Perfect Fluid notion of GR, to Metric-Affine Gravity [2, 3] ? In this direction, many interesting models of fluids with intrinsic hypermomentum have been considered in the past almost 20 years ago (see [4 6] ). However, a generic formulation of an isotropic hyperfluid which represents the direct generalization of the Perfect Fluid was missing so far. Such an attempt was considered recently in [7]. However, that model was tailored only for Cosmological settings and was also laid on the assumption of equivalence between the canonical and the metrical energy momentum tensors of matter. It is the purpose of this note to further extend this consideration by dropping the latter postulate along with homogeneity assumption in order to formulate a generalized isotropic hyperfluid. 
The paper is organized as follows. Firstly, setup our conventions and briefly discuss the basic concepts of a nonRiemannian Geometry[8]. Then we touch upon the sources of MAG, being the canonical energy momentum, the metrical energy momentum and the hypermomentum tensor, and also present their associated conservation laws. Subsequently we formulate the concept of the Perfect (Ideal) Hyperfluid by first giving its physical definition and later using the appropriate mathematical formulation in order to extract its energy tensors by demanding spatial isotropy. Continuing, we apply the conservation laws of MAG to the energy tensors we derived establishing, therefore, a complete description of the novel fluid model we propose. Finally, we consider the Cosmological application of our Hyperfluid model and classify the possible Perfect Cosmological Hyperfluids one can have depending on the equations of state among the hyperfluid variables.

\section{THE SETUP}

We shall now briefly discuss some basic aspects of non-Riemannian geometry that are essential for our analysis. We will use the conventions of [3, 7] so we will go through them rather quickly here.

Let us first fix notation. We shall use letters from the Latin alphabet $a, b, c, \ldots$ to denote anholonomic indices and Greek $\mu, \nu, \rho, \ldots$ for holonomic ones (coordinate) both ranging from $0,1,2, \ldots n-1$ where $n$ is the spacetime dimension. We consider an $n$-dim non-Riemannian manifold endowed with a metric along with a linear connection $(\mathcal{M}, g, \nabla)$. As usual, at each point $p$ on the manifold we can define a set of local frames $\left\{e_{a}\right\}$ spanning the tangent vector space $\mathcal{T}_{p} \mathcal{M}$ of the manifold at that point. We also define the coframe one forms $\left\{\vartheta^{a}\right\}$, living in the cotangent space $\mathcal{T}_{p}^{\star} \mathcal{M}$, through the duality relation

$$
\left.e_{a}\right\rfloor \vartheta^{b}=\delta_{a}^{b}
$$

where 」denotes the interior product. In addition, we assume the existence of a $G L(n, R)$-valued linear connection 1-form $\Gamma_{b}^{a}$ allowing us to define the gauge exterior gauge covariant derivative on arbitrary tensor valued forms. From the gauge potentials $\left(g_{a b}, \vartheta^{a}, \Gamma_{b}^{a}\right)$ we construct their associated field strengths corresponding to non-metricity, torsion and curvature, according to [2]

$$
\begin{gathered}
\mathcal{Q}_{a b}:=-D g_{a b} \\
\mathcal{T}^{a}:=D \vartheta^{a}=d \vartheta^{a}+\Gamma_{b}^{a} \wedge \vartheta^{b} \\
R_{b}^{a}:=d \Gamma_{b}^{a}+\Gamma^{a}{ }_{c} \wedge \Gamma_{b}^{c}
\end{gathered}
$$

respectively. In the above $D$ represents the exterior gauge covariant derivative, and obviously the non-metricity is an 1form while torsion and curvature are both 2 -forms. Also, we define the invariant volume element as $\mu:=\frac{1}{n !} \epsilon_{a_{1} \ldots a_{n}} \vartheta^{a_{1}} \wedge$ $\ldots \wedge \vartheta^{a_{n}}$ and the Hodge dual for an arbitrary $p$-form $\Psi$ as $\star \Psi:=\frac{1}{(n-p) ! p !} g^{a_{1} c_{1}} \ldots g^{a_{p} c_{p}} \epsilon_{a_{1} \ldots a_{p} b_{1} \ldots b_{n-p}} \Psi_{c_{1} \ldots c_{p}} \vartheta^{b_{1}} \wedge \ldots \wedge \vartheta^{b_{n-p}}$. Now, considering an appropriate local gauge transformation we can set the gauge such that ${ }^{1}$

$$
\partial_{\nu} e_{\mu}^{a}-\Gamma_{\mu \nu}^{\rho} e_{\rho}^{a}+\Gamma_{b \nu}^{a} e_{\mu}^{b}=0
$$

where the expansions $e_{a}=e^{\mu}{ }_{a} \partial_{\mu}, \vartheta^{a}=e_{\mu}{ }^{a} d x^{\mu}, \Gamma^{a}{ }_{b}=\Gamma_{b \mu}^{a} d x^{\mu}$ along with the identity $e_{\mu}{ }^{a} e^{\mu}{ }_{b}=\delta_{a}^{b}$, have been used. The latter relation enables one to switch over to a holonomic description based on a metric $g_{\mu \nu}$ and an independent affine connection $\Gamma_{\mu \nu}^{\lambda}$. In this instance our definitions for non-metricity, torsion and curvature respectively read ${ }^{2}$

$$
\begin{gathered}
Q_{\alpha \mu \nu}=-\nabla_{\alpha} g_{\mu \nu} \\
{S_{\mu \nu}{ }^{\lambda}}:=\Gamma_{[\mu \nu]}^{\lambda}
\end{gathered}
$$

\footnotetext{
${ }^{1}$ See [2] for more details. Note that the conventions we are using here have some slight variations from the ones found there.

${ }^{2}$ In the transition from the anholonomic to the holonomic frame description we have denoted $\mathcal{T}^{c}{ }_{\mu \nu}:=-2 S_{\mu \nu}{ }^{c}$ and $\mathcal{Q}_{a b c}=Q_{b c a}$. Note also that the form indices appear on the very right of the component expansion of each object, for instance $\mathcal{Q}_{a b}:=\mathcal{Q}_{a b c} \vartheta^{c}$ and $\mathcal{T}^{a}=\frac{1}{2} \mathcal{T}_{b c}^{a} \vartheta^{a} \wedge \vartheta^{c}$.
} 


$$
R_{\nu \alpha \beta}^{\mu}:=2 \partial_{[\alpha} \Gamma_{|\nu| \beta]}^{\mu}+2 \Gamma_{\rho[\alpha}^{\mu} \Gamma_{|\nu| \beta]}^{\rho}
$$

Our definitions for the associated torsion and non-metricity vectors are

$$
\begin{gathered}
S_{\mu}:=S_{\mu \lambda}^{\lambda}, \quad \tilde{S}_{\mu}:=\epsilon_{\mu \alpha \beta \gamma} S^{\alpha \beta \gamma} \\
Q_{\alpha}:=Q_{\alpha \mu \nu} g^{\mu \nu}, \quad \tilde{Q}_{\nu}=Q_{\alpha \mu \nu} g^{\alpha \mu}
\end{gathered}
$$

respectively. In addition, without the use of any metric, from the Riemann tensor we can derive the two contractions

$$
R_{\nu \beta}:=R_{\nu \mu \beta}^{\mu}, \widehat{R}_{\alpha \beta}:=R_{\mu \alpha \beta}^{\mu}
$$

the first one defines, as usual, the Ricci tensor (which is not symmetric now) and the second one is the homothetic curvature. If a metric is given another contraction can be formed which reads

$$
\breve{R}_{\kappa}^{\lambda}:=R_{\mu \nu \kappa}^{\lambda} g^{\mu \nu}
$$

Note however that the Ricci scalar is always uniquely defined since

$$
R:=R_{\mu \nu} g^{\mu \nu}=-\breve{R}_{\mu \nu} g^{\mu \nu} \quad, \quad \widehat{R}_{\alpha \beta} g^{\mu \nu}=0
$$

Finally, the affine connection can always be decomposed into a Riemannian piece (Levi-Civita connection) plus post Riemannian contributions, according to [3, 9]

$$
\Gamma_{\mu \nu}^{\lambda}=\widetilde{\Gamma}_{\mu \nu}^{\lambda}+N_{\mu \nu}^{\lambda}
$$

where

$$
N_{\alpha \mu \nu}=\frac{1}{2}\left(Q_{\mu \nu \alpha}+Q_{\nu \alpha \mu}-Q_{\alpha \mu \nu}\right)-\left(S_{\alpha \mu \nu}+S_{\alpha \nu \mu}-S_{\mu \nu \alpha}\right)
$$

is the so-called distortion tensor and $\widetilde{\Gamma}_{\mu \nu}^{\lambda}$ is the usual Levi-Civita connection given by

$$
\widetilde{\Gamma}_{\mu \nu}^{\lambda}:=\frac{1}{2} g^{\alpha \lambda}\left(\partial_{\mu} g_{\nu \alpha}+\partial_{\nu} g_{\alpha \mu}-\partial_{\alpha} g_{\mu \nu}\right)
$$

From now on, all Riemannian quantities (i.e. evaluated with respect to the Levi-Civita connection) will be denoted by a tilde. We have now briefly developed the geometric setup to be used for the rest of our analysis. For en extended exposure on the aspects of non-Riemannian geometry the reader is referred to [8].

\section{THE SOURCES OF METRIC-AFFINE GRAVITY (MAG)}

\section{A. Canonical and Metrical Energy Momentum and Hypermomentum Tensors}

As we have already pointed out in the MAG framework one starts with the three independent fields $g_{a b}, \vartheta^{b}$ and $\Gamma_{b}^{a}$. The field equations of a given MAG Theory are obtained by varying the total action independently with respect to those fields. Then, the variations of the matter part of the actions would be the sources of Gravity. Let $\mathcal{L}_{m}$ be the matter Lagrangian of the Theory. Then we have the following variations:

Canonical (Noether) Energy Momentum $(n-1)$-form

$$
\Sigma_{a}:=\frac{\delta \mathcal{L}_{m}}{\delta \vartheta^{a}}
$$

Metrical (Hilbert) Energy Momentum $n$-form

$$
\sigma^{a b}:=2 \frac{\delta \mathcal{L}_{m}}{\delta g_{a b}}
$$


$\underline{\text { Hypermomentum }(n-1) \text {-form }}$

$$
\Delta_{a}{ }^{b}:=-2 \frac{\delta \mathcal{L}_{m}}{\delta \Gamma^{a}{ }_{b}}
$$

Therefore, the three sources of MAG are the canonical, the metrical and the hypermomentum currents of matter [10, 11]. Note that the hypermomentum can be split into its three irreducible pieces of spin, dilation and shear according to

$$
\Delta_{a b}=\tau_{a b}+\frac{1}{n} \Delta g_{a b}+\hat{\Delta}_{a b}
$$

where $\tau_{a b}:=\Delta_{[a b]}$ is the spin part $\Delta:=\Delta_{c d} g^{c d}$ the dilation (trace) and $\hat{\Delta}_{a b}$ the shear (symmetric traceless part). Of course, the physical role of spin and dilation is well known. The most elusive so far has been the role of shear. There have been some interesting early attempts to connect it to the hadronic properties of matter [12] but this connection is not totally clear. For a recent study on the role of shear hypermomentum in Cosmology, see [13]. In a holonomic frame the above canonical, metrical energy momentum and hypermomentum tensors read

$$
\begin{aligned}
& t^{\mu}{ }_{c}=\frac{1}{\sqrt{-g}} \frac{\delta S_{M}}{\delta e_{\mu}{ }^{c}} \\
& T^{\alpha \beta}:=+\frac{2}{\sqrt{-g}} \frac{\delta\left(\sqrt{-g} \mathcal{L}_{M}\right)}{\delta g_{\alpha \beta}} \\
& \Delta_{\lambda}{ }^{\mu \nu}:=-\frac{2}{\sqrt{-g}} \frac{\delta\left(\sqrt{-g} \mathcal{L}_{M}\right)}{\delta \Gamma^{\lambda}{ }_{\mu \nu}}
\end{aligned}
$$

where we have made the identifications $\left.T_{a b}=-\star \sigma_{a b}, t_{a b}=e_{b}\right\rfloor \star \Sigma_{a}$ and $\left.\Delta_{a}{ }^{b d}:=g^{c d} e_{c}\right\rfloor \star \Delta_{a}{ }^{b}$ in order to extract the tensor components. Next we discuss the conservation laws these sources must obey.

\section{B. Conservation Laws}

As we have discussed in the previous section, the sources of MAG are the canonical energy momentum tensor along with the hypermomentum current of matter. Of course there is also the metrical energy momentum tensor but this can be seen as a byproduct of the latter two [2]. These sources are not quite independent and obey certain conservation laws as generalized versions of the energy momentum conservation in GR. Indeed, working in the exterior calculus language, the diffeomorphism invariance of the matter part of the action gives, on-shell $\left[2,[14]^{3}\right.$

$$
\left.\left.\left.D \Sigma_{a}=\frac{1}{2}\left(e_{a}\right\rfloor R_{c}^{b}\right) \wedge \Delta_{b}{ }^{c}-\frac{1}{2}\left(e_{a}\right\rfloor \mathcal{Q}_{b c}\right) \sigma^{b c}+\left(e_{a}\right\rfloor \mathcal{T}^{b}\right) \wedge \Sigma_{b}
$$

In addition, we now also have $G L(n, R)$ invariance which when applied to the matter part implies (still on-shell)

$$
D \Delta_{a}{ }^{b}=2\left(\vartheta^{b} \wedge \Sigma_{a}-\sigma^{b}{ }_{a}\right)
$$

The above two equations give the set of conservation laws which have to be obeyed by the matter sources of MAG. Switching to a holonomic frame, they read [7, 15]

$$
\begin{gathered}
{t^{\mu}}_{\lambda}=T_{\lambda}^{\mu}-\frac{1}{2 \sqrt{-g}} \hat{\nabla}_{\nu}\left(\sqrt{-g} \Delta_{\lambda}{ }^{\mu \nu}\right) \\
\frac{1}{\sqrt{-g}} \hat{\nabla}_{\mu}\left(\sqrt{-g} t_{\alpha}^{\mu}\right)=-\frac{1}{2} \Delta^{\lambda \mu \nu} R_{\lambda \mu \nu \alpha}+\frac{1}{2} Q_{\alpha \mu \nu} T^{\mu \nu}+2 S_{\alpha \mu \nu} t^{\mu \nu}
\end{gathered}
$$

\footnotetext{
3 Translated here to our conventions.
} 
where

$$
\hat{\nabla}_{\nu}:=2 S_{\nu}-\nabla_{\nu}
$$

The above are the MAG conservation laws in the holonomic description. For the most part we will be using this coordinate based formalism (holonomic), which makes things more transparent, but we will give the construction equations of our Perfect Hyperfluid Model in both languages. Again, the set of (26)-(27) or equivalently (24)-(25) has to be obeyed by all matter types of MAG and will be crucial in developing our Perfect Hyperfluid Model.

\section{PERFECT HYPERFLUID: FOUNDATION}

We shall now define the Perfect Hyperfluid as a direct generalization of the Perfect fluid of GR, where now the microstructure of matter (hypermomentum) is also taken into account. Our definition for Perfect Hyperfluid goes as follows.

Definition 1 Let $t_{\mu \nu}, T_{\mu \nu}$ and $\Delta_{\alpha \mu \nu}$ represent the canonical and metrical energy momentum tensors and $\Delta_{\alpha \mu \nu}$ the hypermomentum of the fluid. We define the Perfect Hyperfluid as exactly this fluid whose associated energy tensors $(t, T, \Delta)$ respect spatial isotropy ${ }^{4}$. Mathematically, we demand a vanishing Lie derivative along the spatial slices for each (see also [17]), i.e.

$$
£_{\xi} t_{\mu \nu}=0, £_{\xi} T_{\mu \nu}=0, £_{\xi} \Delta_{\alpha \mu \nu}=0
$$

This implies that both the canonical and the metrical energy momentum tensors would have the perfect fluid form ${ }^{5}$

$$
\begin{gathered}
t_{\mu \nu}=\rho_{c} u_{\mu} u_{\nu}+p_{c} h_{\mu \nu} \\
T_{\mu \nu}=\rho u_{\mu} u_{\nu}+p h_{\mu \nu}
\end{gathered}
$$

where $\rho_{c}, p_{c}$ are the density and pressure associated with the canonical part and $\rho, p$ the usual ones associated to $T_{\mu \nu}$. In addition, demanding only spatial isotropy the hypermomentum takes the covariant form [7]

$$
\Delta_{\alpha \mu \nu}^{(n)}=\phi h_{\mu \alpha} u_{\nu}+\chi h_{\nu \alpha} u_{\mu}+\psi u_{\alpha} h_{\mu \nu}+\omega u_{\alpha} u_{\mu} u_{\nu}+\delta_{n, 4} \epsilon_{\alpha \mu \nu \kappa} u^{\kappa} \zeta
$$

this is the most general covariant form of a type $(0,3)$ tensor respecting isotropy. In the above $\delta_{n, 4}$ is the Kronecker's delta. Note that since we have not imposed homogeneity here, in contrast to [7], all the functions of the set $V=$ $\left\{\rho_{c}, p_{c}, \ldots \omega, \zeta\right\}$ will be generic spacetime functions i.e. $\psi=\psi\left(x^{\alpha}\right)=\psi(t, \vec{x})$ etc. In a covariant fashion, these read

$$
\begin{gathered}
\omega=-u^{\alpha} u^{\mu} u^{\nu} \Delta_{\alpha \mu \nu} \\
\phi=-\frac{1}{(n-1)} h^{\alpha \mu} u^{\nu} \Delta_{\alpha \mu \nu} \\
\chi=-\frac{1}{(n-1)} h^{\alpha \nu} u^{\mu} \Delta_{\alpha \mu \nu} \\
\psi=-\frac{1}{(n-1)} h^{\mu \nu} u^{\alpha} \Delta_{\alpha \mu \nu} \\
\zeta=+\frac{1}{6} \epsilon^{\alpha \mu \nu \lambda} \Delta_{\alpha \mu \nu} u_{\lambda} \delta_{n, 4}
\end{gathered}
$$

\footnotetext{
${ }^{4}$ In other words, following Weinberg's definition [16], in our case' A perfect hyperfluid is defined as this fluid where there exists a velocity $\vec{v}$ such that an observer moving with this velocity will see their surroundings as isotropic'. I am very grateful to Jose Beltran Jimenez for bringing this definition to my attention.

${ }^{5}$ Here, as usual, we consider a normalized velocity field $u_{\mu} u^{\mu}=-1$ and perform an $1+(n-1)$ spacetime split with the projection tensor $h_{\mu \nu}=g_{\mu \nu}+u_{\mu} u_{\nu}$.
} 
These are the 5 material variables describing the hypermomentum part of the fluid. These five fields are then rearranged in a certain way and provide the spin, dilation and shear parts according to

$$
\begin{gathered}
\Delta_{[\alpha \mu] \nu}=(\psi-\chi) u_{[\alpha} h_{\mu] \nu}+\delta_{n, 4} \epsilon_{\alpha \mu \nu \kappa} u^{\kappa} \zeta \\
\Delta_{\nu}:=\Delta_{\alpha \mu \nu} g^{\alpha \mu}=[(n-1) \phi-\omega] u_{\nu} \\
\breve{\Delta}_{\alpha \mu \nu}=\Delta_{(\alpha \mu) \nu}-\frac{1}{n} g_{\alpha \mu} \Delta_{\nu}=\frac{(\phi+\omega)}{n}\left[h_{\alpha \mu}+(n-1) u_{\alpha} u_{\mu}\right] u_{\nu}+(\psi+\chi) u_{(\mu} h_{\alpha) \nu}
\end{gathered}
$$

Let us stress again that all the fields appearing above are generic spacetime functions.

\section{A. Conservation Laws of the Perfect Hyperfluid}

We are now in a position to derive the conservation laws that hold true for our Perfect Hyperfluid Model. As we discussed earlier, in this case the canonical energy momentum tensor has the perfect fluid form and it is therefore symmetric as seen from (45). Then, for any symmetric rank-2 tensor $C_{\mu \nu}$ we have the identity

$$
-\frac{1}{\sqrt{-g}} \hat{\nabla}_{\mu}\left(\sqrt{-g} C^{\mu}{ }_{\nu}\right)=\tilde{\nabla}_{\mu} C^{\mu}{ }_{\nu}-\frac{1}{2} Q_{\nu \alpha \beta} C^{\alpha \beta}-2 S_{\nu \alpha \beta} C^{\alpha \beta}
$$

which can be proved trivially by expanding the left-hand side. Applying this to the canonical energy momentum tensor, the conservation law (27) reads

$$
\tilde{\nabla}_{\mu} t_{\alpha}^{\mu}=\frac{1}{2} \Delta^{\lambda \mu \nu} R_{\lambda \mu \nu \alpha}+\frac{1}{2} Q_{\alpha \mu \nu}\left(t^{\mu \nu}-T^{\mu \nu}\right)
$$

which is also supplemented by

$$
t_{\lambda}^{\mu}=T_{\lambda}^{\mu}-\frac{1}{2 \sqrt{-g}} \hat{\nabla}_{\nu}\left(\sqrt{-g} \Delta_{\lambda}{ }^{\mu \nu}\right)
$$

From the first one above we see that the difference of the canonical and the metrical tensors couples directly to spacetime non-metricity. Of course in the case of the Perfect Cosmological Hyperfluid of [7] this term drops out since in that case the latter two tensors coincide. Note also that the fact that in general the metrical is not the same with the canonical implies that the hypermomentum is not conserved, as seen from (49). In addition, as it is obvious from the above relations one may eliminate the difference of the two energy tensors from (48) by using (49). Then we get the alternative expression

$$
\tilde{\nabla}_{\mu} t_{\alpha}^{\mu}=\frac{1}{2} \Delta^{\lambda \mu \nu} R_{\lambda \mu \nu \alpha}-\frac{1}{4 \sqrt{-g}} Q_{\alpha \mu}{ }^{\lambda} \hat{\nabla}_{\nu}\left(\sqrt{-g} \Delta_{\lambda}{ }^{\mu \nu}\right)
$$

The advantage of this last expression is that it involves only the canonical and the hypermomentum tensors and not the metrical which is a byproduct of the latter two, as seen from (49). Let us collect all the above results and postulate the Perfect Hyperfluid concept along with its complete mathematical description.

Definition 2 The Perfect Hyperfluid: There exists a Perfect (ideal) Hyperfluid structure, carrying intrinsic hypermomentum, that generalizes the Perfect Fluid concept. The description of the Perfect Hyperfluid is given by the energy tensors

$$
\begin{gathered}
t_{\mu \nu}=\rho_{c} u_{\mu} u_{\nu}+p_{c} h_{\mu \nu} \\
T_{\mu \nu}=\rho u_{\mu} u_{\nu}+p h_{\mu \nu} \\
\Delta_{\alpha \mu \nu}^{(n)}=\phi h_{\mu \alpha} u_{\nu}+\chi h_{\nu \alpha} u_{\mu}+\psi u_{\alpha} h_{\mu \nu}+\omega u_{\alpha} u_{\mu} u_{\nu}+\delta_{n, 4} \epsilon_{\alpha \mu \nu \kappa} u^{\kappa} \zeta
\end{gathered}
$$


subject to the conservation laws

$$
\begin{gathered}
\tilde{\nabla}_{\mu} t_{\alpha}^{\mu}=\frac{1}{2} \Delta^{\lambda \mu \nu} R_{\lambda \mu \nu \alpha}+\frac{1}{2} Q_{\alpha \mu \nu}\left(t^{\mu \nu}-T^{\mu \nu}\right) \\
t_{\lambda}^{\mu}=T_{\lambda}^{\mu}-\frac{1}{2 \sqrt{-g}} \hat{\nabla}_{\nu}\left(\sqrt{-g} \Delta_{\lambda}{ }^{\mu \nu}\right)
\end{gathered}
$$

providing a direct generalization of the Perfect Fluid continuum when the intrinsic characteristics of matter (i.e. $\left.\Delta_{\alpha \mu \nu}\right)$ are also taken into account.

Comment: As seen from the above, the complete description of the Perfect Hyperfluid is given by the set of 9 spacetime functions $\left\{\rho, \rho_{c}, p, p_{c}, \phi, \chi, \psi, \omega, \zeta\right\}$ along with its associated velocity field $u$.

\section{B. Exterior Calculus representation of Perfect Hyperfluid}

For completeness we will give the forms of energy tensors of the Perfect Hyperfluid (MAG sources) in the language of exterior differential forms, which is of great use in MAG. It can be easily seen that the hypermomentum expression (47), in the language of differential forms, translates to the hypermomentum $(n-1)$ form

$$
\left.\left.\left.\left.\Delta_{a}{ }^{b}=\delta_{a}^{b} \phi u+\chi\left(e^{b}\right\rfloor \star u\right) \star \vartheta_{a}+\psi\left(e_{a}\right\rfloor \star u\right) \star \vartheta^{b}+\left(e_{a}\right\rfloor \star u\right)\left(e^{b}\right\rfloor \star u\right) \bar{\omega} u-3 ! \vartheta_{a} \wedge \vartheta^{b} \wedge Z \delta_{n, 4}
$$

where $\phi, \chi, \psi$ and $\bar{\omega}=\omega+\phi+\chi+\psi$ are 0 -forms and $Z=\zeta_{a} \vartheta^{a}=\zeta u_{a} \vartheta^{a}=\zeta \star u$ is an 1-form. The associated canonical $(n-1)$-from and metrical $n$-form currents are also extracted rather trivially and read

$$
\begin{gathered}
\left.\Sigma_{a}=(\bar{\rho}+\bar{p})\left(e_{a}\right\rfloor \star u\right) u+\bar{p} \star \vartheta_{a} \\
\sigma_{a b}=\mu\left((\rho+p) u_{a} u_{b}+p g_{a b}\right)
\end{gathered}
$$

respectively. In the above with have denoted $\rho_{c}=\bar{p}$ and $p_{c}=\bar{p}$ in order to avoid confusion with the anholonomic index $c$. In addition, we have considered the flow $(n-1)$-form (see for instance [5]) $u$ with its dual giving us the velocity field

$$
\star u:=u_{a} \vartheta^{a}
$$

and the normalization

$$
u \wedge \star u=\mu
$$

With this we may re-express (50) in the more transparent form

$$
\Delta_{a}{ }^{b}=\delta_{a}^{b} \phi u+\chi u^{b}\left(\star \vartheta_{a}\right)+\psi u_{a}\left(\star \vartheta^{b}\right)+u_{a} u^{b} \tilde{\omega} u-3 ! \zeta \vartheta_{a} \wedge \vartheta^{b} \wedge(\star u) \delta_{n, 4}
$$

The latter expression along with (51) and (52) represent the material sources of the Perfect (Ideal) Hyperfluid in the language of differential forms and are subject to the conservation laws (24) and (25).

\section{THEORIES WITH $\mathcal{L}_{G}=\mathcal{L}_{G}\left(R^{\lambda}{ }_{\alpha \beta \gamma}\right)$}

In the special case of Theories whose Gravitational part is constructed only by the Riemann tensor and its contractions we have a very important restriction on the Hyperfluid sector. Indeed, as can be trivially checked in this case, the Riemann tensor is invariant under special projective transformations of the form ${ }^{6}$

$$
\Gamma_{\mu \nu}^{\lambda} \longrightarrow \bar{\Gamma}_{\mu \nu}^{\lambda}=\Gamma_{\mu \nu}^{\lambda}+\delta_{\mu}^{\lambda} \partial_{\nu} \lambda
$$

\footnotetext{
${ }^{6}$ Also known as $\lambda$-transformations.
} 


$$
R_{\mu \nu \alpha}^{\lambda} \longrightarrow \bar{R}_{\mu \nu \alpha}^{\lambda}=R_{\mu \nu \alpha}^{\lambda}
$$

Of course, all contractions of the Riemann tensor will also respect this symmetry. The above fact has a great impact on the hypermomentum sources. Indeed, since this symmetry have to be respected from the matter part as well, we get the constraint (see for instance [18])

$$
\partial_{\nu}\left(\sqrt{-g} \Delta^{\nu}\right)=0
$$

that is, the dilation current $\Delta^{\mu}:=\Delta_{\lambda}{ }^{\lambda \nu}$ must be conserved. Equivalently, taking the contraction of (49) the above constraint translates to

$$
t=T
$$

namely the traces of the canonical and the metrical tensors must coincide. Note that up to now we have made no assumption on the matter content of the Theory. If we apply the above result to our Perfect Hyperfluid model, given the forms (45) and (46) it follows that

$$
-\rho_{c}+(n-1) p_{c}=-\rho+(n-1) p
$$

In addition, assuming that both perfect fluid components (metrical and canonical) are barotropic, that is $p_{c}=w_{c} \rho_{c}$ and $p=w \rho$, we have

$$
\rho_{c}=\left(\frac{1-(n-1) w}{1-(n-1) w_{c}}\right) \rho
$$

From the above discussion it is now clear that if the barotropic components of the canonical and the metrical are of the same kind, in the sense that $w=w_{c}$ we immediately have that $p=p_{c}$ and from (61) it follows that $\rho=\rho_{c}$ as well. Conversely, on the (logical!) assumption that the associated densities are equal (i.e. $\rho=\rho_{c}$ ) we also have that $p=p_{c}$ from the above equation. Either way the end result is that both the pressures and the densities would be identical and consequently $t_{\mu \nu} \equiv T_{\mu \nu}$. We therefore see how the Perfect Cosmological Hyperfluid Model of [7] is embedded in our general construction here. Given that the Gravitation part is built only from the Riemann tensor and its contractions, the Perfect Hyperfluid of [7] is the one for which the barotropic fluid components are of the same kind $w=w_{c}$ or alternatively the canonical and metrical densities coincide. Recall that in this case the conservation laws take the form

$$
\begin{gathered}
\widetilde{\nabla}_{\mu} T_{\nu}^{\mu}=\frac{1}{2} \Delta^{\alpha \beta \gamma} R_{\alpha \beta \gamma \nu} \\
\hat{\nabla}_{\nu}\left(\sqrt{-g} \Delta_{\lambda}{ }^{\mu \nu}\right)=0, \quad t_{\mu \nu}=T_{\mu \nu}
\end{gathered}
$$

For this reason we shall call this Model the Hypermomentum Conserving Perfect Hyperfluid. The above considerations were rather general with no assumption about the underlying spacetime. If we consider a Cosmological setting the fluid obeying the above two conservation laws (that is, the fluid in [7]) will be called the Hypermomentum Preserving Perfect Cosmological Hyperfluid.

\section{COSMOLOGICAL APPLICATION OF THE PERFECT HYPERFLUID}

Let uf now see an immediate application of our Fluid model. If we now impose also homogeneity and consider and FLRW Universe all the variables, of the hyperfluid, would be time dependent only and in this case our conservation laws (48) and (49) boil down to ${ }^{7}$

$$
[\dot{\rho}+(n-1) H(\rho+p)] u_{\nu}+(\rho+p) u^{\mu} \widetilde{\nabla}_{\mu} u_{\nu}=\frac{1}{2} u^{\mu}\left(\phi \widehat{R}_{\mu \nu}+\chi R_{\mu \nu}+\psi \breve{R}_{\mu \nu}\right)+\frac{1}{2} u_{\nu}\left[\left(\rho_{c}-\rho\right) C+\left(p_{c}-p\right) A\right]
$$

\footnotetext{
${ }^{7}$ As usual, the dot denotes time derivative.
} 


$$
\begin{gathered}
-\delta_{\lambda}^{\mu} \frac{\partial_{\nu}\left(\sqrt{-g} \phi u^{\nu}\right)}{\sqrt{-g}}-u^{\mu} u_{\lambda} \frac{\partial_{\nu}\left(\sqrt{-g}(\phi+\chi+\psi+\omega) u^{\nu}\right)}{\sqrt{-g}} \\
+\left[\left(2 S_{\lambda}+\frac{Q_{\lambda}}{2}\right) u^{\mu}-\nabla_{\lambda} u^{\mu}\right] \chi+\left[\left(2 S^{\mu}+\frac{Q^{\mu}}{2}-\tilde{Q}^{\mu}\right) u_{\lambda}-g^{\mu \nu} \nabla_{\nu} u_{\lambda}\right] \psi \\
+u^{\mu} u_{\lambda}(\dot{\chi}+\dot{\psi})-(\phi+\chi+\psi+\omega)\left(\dot{u}^{\mu} u_{\lambda}+u^{\mu} \dot{u}_{\lambda}\right)=2\left(\rho-\rho_{c}\right) u_{\lambda} u^{\mu}+2\left(p-p_{c}\right) h_{\lambda}{ }^{\mu}+
\end{gathered}
$$

The above equations contain the full dynamics of the generalized Perfect Cosmological Hyperfluid. Let us highlight again that the degrees of freedom in this case $\operatorname{are}^{8} 2+2+5=9$. However, due to the high symmetry of FLRW spacetime, the above conservation laws only provide $1+2$ evolution equations. This is no surprise since a similar situation appears in the case of the Perfect Fluid of GR where the continuity equation only gives the evolution for $\rho$ and $\mathrm{p}$ is usually assumed to satisfy an equation of state $p=w \rho$ such that the system becomes complete. The same behaviour we also expect here, so we will need to provide two barotropic indices for the metrical and canonical parts according to

$$
p=w \rho, \quad p_{c}=w_{c} \rho_{c}
$$

In addition, there should be one equation relating the above 4 functions (similar to (61)) and we should also have three equations of state among the hypermomentum variables, in order to have a completely determined dynamics. In general, the three equations of state among the hypermomentum variables would have the form

$$
F_{A}(\phi, \chi, \psi, \omega, \zeta)=0, \quad A=1,2,3
$$

To further restrict the above possibility it would be most natural to associate equations of state among the different parts of hypermomentum (spin, dilation and shear) as was implemented in [13]. The exact values of these equations of state would characterize the nature of the hyperfluid (see also 7] where such equations of state are derived). We should also note that it would be possible to have equations of state that mix up the perfect fluid with the hypermomentum parts. Then, one needs 6 independent equations of state, whose generic form would read

$$
F_{I}\left(\rho, p, \rho_{c}, p_{c}, \phi, \chi, \psi, \omega, \zeta\right)=0, \quad I=1,2, \ldots, 6
$$

As a result, the Perfect Cosmological Hyperfluid lies in the intersection of the aforementioned 6 hypersurfaces. However, we find the latter mixing possibility very unlikely, though it may be true for some types of generalized hyperfluids. In any case, the three conservation laws for any type of Cosmological Hyperfluids, can be extracted from (64) and (65) by first contracting the former with $u^{\mu}$ and take from the latter one time the $i j$-components and another the 00 to arrive at

$$
\begin{gathered}
\dot{\rho}_{c}+(n-1) H\left(\rho_{c}+p_{c}\right)=-\frac{1}{2} u^{\mu} u^{\nu}\left(\chi R_{\mu \nu}+\psi \breve{R}_{\mu \nu}\right)+\frac{1}{2}\left(\rho_{c}-\rho\right) C+\frac{1}{2}\left(p_{c}-p\right) A \\
\dot{\phi}+(n-1) H \phi+H(\chi+\psi)+\psi X-\chi Y=2\left(p_{c}-p\right) \\
\dot{\omega}+(n-1) H(\chi+\psi+\omega)+(n-1)(\psi X-\chi Y)=2\left(\rho_{c}-\rho\right)
\end{gathered}
$$

Additionally, one could take the trace of (65) to arrive at

$$
(n-1) \dot{\phi}-\dot{\omega}+(n-1) H[(n-1) \phi-\omega]=2\left[\left(\rho-\rho_{c}\right)-(n-1)\left(p-p_{c}\right)\right]
$$

However, as expected, this gives no further information since it is easily seen that the latter is equal to ${ }^{9}(n-1)(70)-$ (71). Therefore, as we have already mentioned, the full dynamics of the Perfect Hyperfluid is contained in the three equations (69), (70) and (71). We should emphasize again that the latter equations are fairly general and hold true regardless of the equations of state to be imposed on the hyperfluid variables. For any Metric-Affine Cosmology, the evolution equations for the sources are the aforementioned three.

Let us now organize the above ideas according to the following classifications. We start with the most general case and subsequently specialize.

\footnotetext{
8 Obviously two degrees of freedom come from $\rho, p$ another two from $\rho_{c}, p_{c}$ and the rest are the five hypermomentum variables.

9 As is also apparent from (72) the dilation current is conserved only when $\left(\rho-\rho_{c}\right)=(n-1)\left(p-p_{c}\right)$. This condition is always true for frame rescaling invariant Theories [18].
} 


\section{A. Cosmological Hyperfluids Classification}

Below we classify some characteristic cases of the Perfect Cosmological Hyperfluids.

Definition 3 A generalized Perfect Cosmological Hyperfluid consists of a set of energy tensors given by the expressions (45), (46) and (47) subject to the conservation laws

$$
\begin{gathered}
\tilde{\nabla}_{\mu} t_{\alpha}^{\mu}=\frac{1}{2} \Delta^{\lambda \mu \nu} R_{\lambda \mu \nu \alpha}+\frac{1}{2} Q_{\alpha \mu \nu}\left(t^{\mu \nu}-T^{\mu \nu}\right) \\
t_{\lambda}^{\mu}=T_{\lambda}^{\mu}-\frac{1}{2 \sqrt{-g}} \hat{\nabla}_{\nu}\left(\sqrt{-g} \Delta_{\lambda}{ }^{\mu \nu}\right)
\end{gathered}
$$

In addition, its 9 material sources are related to one another by the 6 generalized equations of state

$$
F_{I}\left(\phi, \chi, \psi, \omega, \zeta, \rho, p, \rho_{c}, p_{c}\right)=0, \quad I=1,2, \ldots, 6
$$

Definition 4 A Barotropic Perfect Cosmological Hyperfluid represents a special case of the above for which the equations of state (75) have the barotropic form

$$
\sum_{i=1}^{9} a_{i}^{I} X_{i}=0, I=1,2, \ldots, 6, \quad i=1,2, \ldots, 9
$$

where not all $a_{i}^{I}$ 's are zero and we have also collectively denoted $X_{i}=\left\{\rho_{c}, p_{c}, \rho, p, \phi, \chi, \psi, \omega, \zeta\right\}$.

Definition 5 A Decoupled Barotropic Perfect Cosmological Hyperfluid is the one for which the barotropic equations among its constituents have the following forms

$$
\begin{gathered}
p=w \rho, \quad p_{c}=w_{c} p_{c}, \quad, \rho_{c}=\bar{w} \rho \\
\sum_{i=1}^{6} a_{i}^{I} Y_{i}=0, I=1,2, \ldots, 6, \quad i=1,2, \ldots, 5
\end{gathered}
$$

where not all $a_{i}^{I}$ 's are zero and we have collectively denoted $Y_{i}=\{\phi, \chi, \psi, \omega, \zeta\}$.

Comment: The Perfect Hypermomentum Preserving Cosmological Hyperfluid (see previous section) represents a special case of the latter with $\rho_{c}=\rho$ and $p_{c}=p$.

\section{DISCUSSION}

We have constructed a straight generalization of the familiar Perfect Fluid notion, encompassing now the microscopic characteristics of matter as well. We call this extended fluid notion a Perfect (Ideal) Hyperfluid. The latter is described by the three tensors of the canonical, metrical and hypermomentum currents, all of which have an isotropic form (see eqn's (45)-(47)) and are subject to the MAG conservation laws. As soon as the intrinsic properties of the fluid are neglected $\left(\Delta_{\alpha \mu \nu}=0\right)$ we arrive at the classical Perfect Fluid model as a limiting case, as expected. The description of the generalized Perfect Hyperfluid is given by 9 spacetime functions along with the fluids' velocity flow $u$. The specific form of these 9 functions will characterize the fluid under consideration.

It should be noted that even though our generalized Perfect Hyperfluid construction fits most naturally in a MetricAffine Gravity approach, this is by no means the only place it can find applications. Indeed, our general construction here can just as well be applied to all Theories that represent special cases of MAG. For instance, Einstein-Cartan [19] or more generalized torsionful Theories (like Poincare Gravity [20]), non-metric torsionless Theories, and also to all teleparallel Theories such as metric [21], symmetric [22, 23] and generalized teleparallelism [24]. Of course the list could go on and on. In general we expect the Perfect Hyperfluid to describe matter configurations to all Gravity Theories exhibiting a non-trivial connection.

Furthermore, the Perfect Hyperfluid could find interesting applications in the Theory of materials with microstructure and elasticity [25]. This is plausible since the very concept of hypermomentum has a close analogy with a notion appearing in materials with microstructure, which is known as hyper-stress [26]. Lastly, probably the most obvious application of our hyperfluid structure would be to use it in order to study the full quadratic MAG Theory in a Cosmological setting and in the presence of the latter fluid configuration. This is currently under investigation. 


\section{ACKNOWLEDGMENTS}

I would like to thank Jose Beltran Jimenez and Tomi Sebastian Koivisto for some useful discussions. This research is co-financed by Greece and the European Union (European Social Fund- ESF) through the Operational Programme 'Human Resources Development, Education and Lifelong Learning' in the context of the project "Reinforcement of Postdoctoral Researchers - 2 nd Cycle" (MIS-5033021), implemented by the State Scholarships Foundation (IKY).

[1] Dirk Puetzfeld and Yuri N Obukhov. Probing non-riemannian spacetime geometry. Physics Letters A, 372(45):6711-6716, 2008.

[2] Friedrich W Hehl, J Dermott McCrea, Eckehard W Mielke, and Yuval Ne'eman. Metric-affine gauge theory of gravity: field equations, noether identities, world spinors, and breaking of dilation invariance. Physics Reports, 258(1-2):1-171, 1995.

[3] Damianos Iosifidis. Metric-affine gravity and cosmology/aspects of torsion and non-metricity in gravity theories. arXiv preprint arXiv:1902.09643, 2019.

[4] Yuri N Obukhov and Romualdo Tresguerres. Hyperfluid-a model of classical matter with hypermomentum. Physics Letters A, 184(1):17-22, 1993.

[5] Yuri N Obukhov. On a model of an unconstrained hyperfluid. Physics Letters A, 210(3):163-167, 1996.

[6] Olga V Babourova and Boris N Frolov. Perfect hypermomentum fluid: Variational theory and equations of motion. International Journal of Modern Physics A, 13(31):5391-5407, 1998.

[7] Iosifidis Damianos. Cosmological hyperfluids, torsion and non-metricity. The European Physical Journal. C, Particles and Fields., 80(11), 2020.

[8] Luther Pfahler Eisenhart. Non-riemannian geometry. Courier Corporation, 2012.

[9] JA Schouten. Ricci-calculus. an introduction to tensor analysis and its geometrical applications, 1954.

[10] Friedrich W Hehl, G David Kerlick, and Paul von der Heyde. On hypermomentum in general relativity i. the notion of hypermomentum. Zeitschrift fuer Naturforschung A, 31(2):111-114, 1976.

[11] FW Hehl, EA Lord, and Y Ne'Eman. Hypermomentum in hadron dynamics and in gravitation. Physical Review D, 17(2):428, 1978.

[12] Friedrich W Hehl and Yuri N Obukhov. Is ahadronic'shear current one of the sources in metric-affine gravity? arXiv preprint gr-qc/9712089, 1997.

[13] Damianos Iosifidis. Non-riemannian cosmology: The role of shear hypermomentum. arXiv preprint arXiv:2010.00875, 2020.

[14] Frank Gronwald. Metric-affine gauge theory of gravity: I. fundamental structure and field equations. International Journal of Modern Physics D, 6(03):263-303, 1997.

[15] Yuri N Obukhov and Dirk Puetzfeld. Conservation laws in gravitational theories with general nonminimal coupling. Physical Review D, 87(8):081502, 2013.

[16] Steven Weinberg. Gravitation and cosmology: principles and applications of the general theory of relativity. 1972.

[17] Michael Tsamparlis. Cosmological principle and torsion. Physics Letters A, 75(1-2):27-28, 1979.

[18] Damianos Iosifidis and Tomi Koivisto. Scale transformations in metric-affine geometry. Universe, 5(3):82, 2019.

[19] Andrzej Trautman. Einstein-cartan theory. arXiv preprint gr-qc/0606062, 2006.

[20] Kenji Hayashi and Takeshi Shirafuji. Gravity from poincaré gauge theory of the fundamental particles. i: General formulation. Progress of Theoretical Physics, 64(3):866-882, 1980.

[21] Ruben Aldrovandi and Jose G Pereira. Teleparallel gravity: an introduction, volume 173. Springer Science \& Business Media, 2012.

[22] James M Nester and Hwei-Jang Yo. Symmetric teleparallel general relativity. arXiv preprint gr-qc/9809049, 1998.

[23] Jose Beltrán Jiménez, Lavinia Heisenberg, and Tomi S Koivisto. Teleparallel palatini theories. Journal of Cosmology and Astroparticle Physics, 2018(08):039, 2018.

[24] Jose Beltrán Jiménez, Lavinia Heisenberg, Damianos Iosifidis, Alejandro Jiménez-Cano, and Tomi S Koivisto. General teleparallel quadratic gravity. Physics Letters B, page 135422, 2020.

[25] Raymond David Mindlin. Microstructure in linear elasticity. Technical report, Columbia Univ New York Dept of Civil Engineering and Engineering Mechanics, 1963.

[26] Frank Gronwald and Friedrich W Hehl. Stress and hyperstress as fundamental concepts in continuum mechanics and in relativistic field theory. arXiv preprint gr-qc/9701054, 1997. 